\title{
THE COMBINED EFFECT OF PLATFORM EDGE DOORS AND LEVEL ACCESS ON THE BOARDING AND ALIGHTING PROCESS IN THE LONDON UNDERGROUND
}

\author{
Sebastián Seriani, Corresponding Author \\ University College London \\ Chadwick Building, Gower St., London, WC1E 6BT, United Kingdom \\ Tel: +44-(0)-20-7679-7224; Email: sebastian.seriani.14@ucl.ac.uk \\ Gonzalo de Ana Rodríguez \\ Rail \& Underground Transport Planning \\ Transport for London \\ 197 Blackfriars Road, London, SE1 8NJ, United Kingdom \\ Tel: +44-(0)-20-3054-6431; Email: gonzalodeana@tfl.gov.uk \\ Catherine Holloway \\ University College London \\ Gower St. 66-72, London, WC1E 6BT, United Kingdom \\ Tel: +44-(0)-20-7679-1568; Email: c.holloway@ucl.ac.uk \\ Word count: 5,484 words text +24 references +6 tables/figures $\mathrm{x} 250$ words $=6,084$ words \\ 30 July 2016
}

28 


\section{ABSTRACT}

2 Platform edge doors (PEDs) are used in metro stations to improve passengers' safety and comfort, whilst 3 a step-free access with minimum gap between the train and the platform is desirable on the grounds of 4 accessibility.

There is little research focused on the effect of PEDs on the boarding and alighting time (BAT) and passenger behaviour patterns. On the other hand, many authors have treated the impact of vertical and horizontal gaps in passengers' boarding and alighting.

8 On London Underground (LU), there is always step-free access between the train and the platform when 9 there are PEDs; but even at some platforms without PEDs there may be level access provided by platform humps.

The objective of this paper is to study the combined effect of PEDs and level access on the boarding and alighting process. To this aim two LU platforms, both with level access, one with PEDs and one without PEDs, have been compared. This was done analysing bespoke video footage. The results show that PEDs on their own have no overall negative impact on the BAT and that in most situations they encourage passengers to wait beside the doors. It was also found that demand (number of boarders, alighters, and passengers on the train) is more important on the BAT and passenger behaviours than the presence of PEDs.

Keywords: dwell time; boarding, alighting, passenger behaviour, platform edge door, level access. 


\section{INTRODUCTION}

Platform edge doors (PEDs) are used in metro systems around the world mainly to improve passengers' safety $(1,2,3)$. Currently, the London Underground (LU) network has PEDs in nine stations on the Jubilee Line, all of which provide level access to the trains from the whole platform. However, at many other LU stations, there is a certain gap between the train and the platform. The vertical gap typically varies with the type of rolling stock, whilst the horizontal gap is usually most influenced by the platform design (e.g. curved platforms generate wider horizontal gaps). It is recommended that vertical gaps should not exceed $50 \mathrm{~mm}$, and that horizontal gaps be lower than $75 \mathrm{~mm}(4)$.

To reduce these gaps at the platform train interface (PTI), platform humps have been retrofitted at some stations to provide equal accessibility to all users and ease the boarding and alighting of passengers with mobility impairments or encumbrances such as heavy luggage or buggies. They are located in front of some doors and cover the whole platform width, with gentle access slopes on either side and specific signage. However, the implementation of platform humps is limited by the need of consistency in train stopping and the different types of rolling stock (5).

There is a common presumption that the presence of PEDs increases both the static and dynamic components of dwell time, which is defined as the time that the train remains on a platform from the moment the wheels stop to the time when they start rolling again. The static component of dwell time is related to the door opening and closing times, as well as to the duration of other mechanical movements and of safety delays, whilst the dynamic compoment relates to passenger movements and is mainly the boarding and alighting time (BAT), which is the focus of this paper.

However, recent studies ( 6 ) have shown that PEDs have no important impact on the BAT, although these elements change waiting passengers' behaviour, whereby queuing or clustering beside the doors rather than in front of them happens more often when there are PEDs. For that study, the authors used a mock-up train and PTI in a controlled environment at University College London's Pedestrian Accessibility Movement Environmental Laboratory (PAMELA) and conducted observations from video footage at two LU platforms. For both the experiments and observations in ( 6 ), they compared a PTI with PEDs and level access with another PTI without PEDs and a $170 \mathrm{~mm}$ high vertical gap. However, due to a technical fault in the automatic video recording and lack of resources, the authors could not consider a PTI without PEDs and with level access, therefore the results obtained left the question of the effect of PEDs under equal vertical gap unresolved for further research.

The aim of this work is thus to fill that knowledge gap and to expand the analysis and results obtained in (6), studying the effect of PEDs on the BAT and on passengers' behaviour when level access is provided. In this work behaviour is defined as the way passengers interact with each other at the PTI (7).

This paper comprises six sections, including this one. The second section is the literature review. In the third, the method is explained. The fourth section presents the results. In the fifth, these are further discussed, and finally in the sixth section the conclusions are explained.

\section{LITERATURE REVIEW}

The boarding and alighting time (BAT) has been studied by different authors. Here, only a few contributions are discussed.

The Highway Capacity Manual and Transit Capacity and Level of Service Manual $(8,9)$ propose a linear relationship to determine dwell time. The time to open and close the doors is considered the static component, whilst the dynamic component or BAT is defined as the product of the average time it takes each passenger to board or alight and the number of passenger boarding and alighting. However, some field studies (10) show that the BAT depends not only on the number of passengers boarding and alighting but also on other factors such as the door width, where wider doors may reduce the BAT by up to $10 \%$.

Moreover, other authors $(11,12)$ use the LU Train Service Model to describe the BAT as part of the station stop time, where the BAT is given by a non-linear relationship between the number of passengers boarding and alighting, the number of doors per car, the number of seats per car, the number of through passengers, and two adjustment factors: the peak door/average door factor and the door width factor. They found that wider doors, larger stand-backs, and more seats can reduce the BAT. In particular, the BAT is determined by the total alighting time, total boarding time and total interaction time at the busiest door (11), where the interaction time is defined as the overlap time when boarding and alighting occurs simultaneously at the busiest door. 
According to laboratory experiments, the BAT depends on the vertical gap, door width, fare

\section{METHODS}

The analysis of the boarding and alighting processes is based on observations made on video footage recorded under actual operating conditions at two LU platforms. The Jubilee line southbound platforms at Green Park (GPK) and Westminster (WMS) stations were chosen because of their similarities in terms of demand and platform layout, other than the main PTI difference that was being tested, i.e. the presence of PEDs at WMS versus a PTI without PEDs at GPK.

Since the aim of this study is to analyse the impact of PEDs when the vertical gap is the same, it was necessary to get footage from doors at GPK and WMS which would satisfy this condition. As was pointed earlier, on LU all platforms with PEDs (such as WMS), have level access along their whole length; but this is not the case in GPK. However, GPK has some doors at a platform hump where there is no vertical gap between train and platform. Therefore these were the only doors at GPK that could be used for this study. The platform hump at GPK has a total length of $27 \mathrm{~m}$ and extends over the whole platform width, therefore covering the second and third cars and a total of four doors (two doubles and two singles).

The footage analysed for one of the hump doors at GPK was recorded between 23 November 2015 and 7 December 2015 and comprises only the weekday morning and evening peak 
hours (08:15-09:15 and 17:15-18:15 pm), when trains on that line reach an average frequency of 30 trains/h (approximately 2 minutes' headways).

These videos were compared to the footage from two doors at WMS which was obtained for the previous study (6). Those videos are from November 2014, i.e. at the same time of the year but one year earlier, but it is considered that the differences that could arise because of the year difference are negligible compared to the differences due to the different PTI arrangements (presence of PEDs) and to the demand, which was measured for all boarding and alighting processes in the same way.

In summary, this analysis compares:

- Two double doors at WMS, where there is level access on the whole platform; with

- One double door at GPK, located at the platform hump

At both stations the double doors are $1.60 \mathrm{~m}$ wide and the horizontal gap at the PTI is $90 \mathrm{~mm}$.

To measure the boarding and alighting time (BAT), the number of passengers boarding $\left(\mathrm{P}_{\mathrm{b}}\right)$ and alighting $\left(\mathrm{P}_{\mathrm{a}}\right)$ was manually counted in segments of five seconds from the time the doors opened until they closed or after $120 \mathrm{~s}$, whichever the greatest.

The BAT, $\mathrm{P}_{\mathrm{b}}$ and $\mathrm{P}_{\mathrm{a}}$ were corrected to eliminate the effect of "late runners", i.e. passengers boarding the train after the main group has already boarded. This helps to remove the impact of longer dwells which are to do with the train being held at the platform rather than with passenger movements, which are the focus of this analysis. The criterion used for this correction considers "late runners" those passengers who board or alight after two or more segments $(10 \mathrm{~s})$ in which there are no other movements. After this correction the average overlap time $\left(T_{0}\right)$ was calculated (in $5 \mathrm{~s}$ segments), which is defined as the total time (sum of $5 \mathrm{~s}$ segments) when passengers board and alight simultaneously.

Aside from the presence of PEDs, the demand is considered to have a significant impact on the BAT. Since it was not possible to control the level of demand under actual operation, demand was measured and the observations aggregated with respect to two factors:

- Total number of boarders and alighters;

- Train demand on arrival.

From these factors, only the demand on arrival could not be obtained from the videos. As an alternative, it was obtained from NetMIS, TfL's network management information system, which provides a level of demand (low-medium-high) for each arriving train.

In addition to the BAT analysis, the behaviour of passengers waiting to board the train was also studied. To this aim, two types of behaviour were recorded when trains stopped at the platform:

- Passengers waiting beside the doors;

- Passengers waiting in front of the doors.

It is important to note that these behaviours are not exclusive, i.e in the same boarding and alighting process there may be passengers waiting both in front of and beside the doors (e.g. in crowded situations when there are passengers everywhere around the doors).

Finally, and similarly to the analysis of actual observations in (6), this work is limited to descriptive statistics, without formal statistical significance tests. This is because the data do not satisfy the assumptions of any statistical tests, either parametric (e.g. ANOVA) or non-parametric (e.g. MannWhitney).

\section{RESULTS}

\section{Impact on the BAT}

\section{High level statistics}

Table 1 shows summary statistics of the average BAT and numbers of boarders and alighters at the two stations, with and without PEDs. At face value, the case without PEDs presents an average BAT which is $16 \%$ lower than in the case with PEDs. However, it is difficult to draw simple conclusions about the BAT because it is influenced by demand, and as it can be seen, the case without PEDs (GKP) has an average number of passengers boarding and alighting $\left(\mathrm{P}_{\mathrm{a}}+\mathrm{P}_{\mathrm{b}}\right)$ which is $33 \%$ lower than in WMS, where there are PEDs, and a much different split between boarders and alighters, as given by the different ratios $(\mathrm{R})$. 


\begin{tabular}{llll}
$\begin{array}{l}\text { Variable } \\
\text { (average over observations) }\end{array}$ & WMS (1) & GKP (2) & $\begin{array}{l}\text { Difference to PEDs } \\
\text { (2 with respect to 1) }\end{array}$ \\
\hline BAT (s) & 23.0 & 19.4 & $-16 \%$ \\
$\mathrm{P}_{\mathrm{a}}$ (passengers) & 6 & 5 & $-16 \%$ \\
$\mathrm{P}_{\mathrm{b}}$ (passengers) & 12 & 7 & $-42 \%$ \\
$\mathrm{P}_{\mathrm{a}}+\mathrm{P}_{\mathrm{b}}$ (passengers) & 18 & 12 & $-33 \%$ \\
$\mathrm{R}=\mathrm{P}_{\mathrm{b}} / \mathrm{P}_{\mathrm{a}}$ & 4.8 & 1.8 & $-63 \%$ \\
\hline
\end{tabular}

\section{Boarding and alighting profiles}

Figure 1 shows the average boarding and alighting profiles for each case. They have been constructed as relative profiles to isolate the effect of demand and get the boarding and alighting patterns out of the shape of the curves. To calculate the relative boardings or alightings at each $5 \mathrm{~s}$ segment, the number of boarders or alighters is divided by the total number of boarders and alighters in that boarding and alighting process. But the profiles in Figure 1 are the average of all the profiles so built of all recorded boarding and alighting processes.

It can be seen that the case without PEDs (GPK) has a higher and earlier peak value in the boarding profile as compared to the case with PEDs (WMS). However, even if boarding peaks later in the case of PEDs (probably due to people giving way to alighters), the boarding profiles then converge to zero at almost the same time, so that in both cases most passengers have boarded before $32.5 \mathrm{~s}$. In other words, the earlier peak is compensated by a quicker drop to zero. The largest difference in the cumulative boarding profiles occurs after $12.5 \mathrm{~s}$, where on average $11 \%$ more passengers have boarded at GKP compared to WMS, but this difference fades away at $32.5 \mathrm{~s}$.

The alighting pattern is much more consistent, and a similar cumulative alightings profile could have been plotted (not done for clarity), in which the largest difference between PEDs and no PEDs would have occurred after $7.5 \mathrm{~s}$ and been approximately $14 \%$ more passengers alighted at GKP (no PEDs) compared to WMS (PEDs), but the difference virtually disappears after $12.5 \mathrm{~s}$.

Relative boarding and alighting profiles

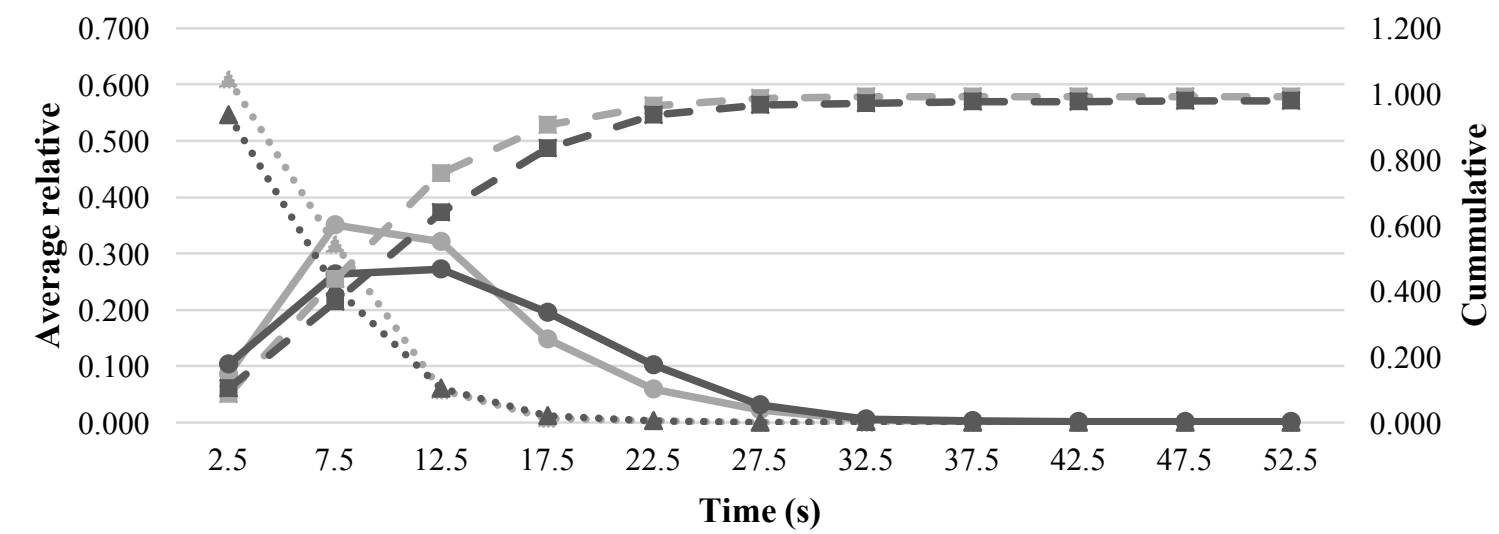

FIGURE 1 Average relative boarding and alighting profiles with (WMS) and without PEDs (GPK).

\section{Demand}

This section looks at the impact of PEDs on BAT for the different demand categories, to decouple their influence on the BAT. 
In relation to the train demand on arrival, Table 2 shows that PEDs seem to increase BAT for medium and high on-train loads. However, there are no "low" loads reported for the hump door (no PEDs). This could be caused because those demand levels are actually not reached, but it is important to insist on the limitations of these data, which firstly are not very accurate in their distinction among low-medium-high and secondly come from a separate dataset which has to be matched to the observations, which could have introduced some mismatch errors. Therefore, any conclusions in this regard should be treated with circumspection.

With respect to the total number of boarders and alighters, Table 2 shows that the BAT increases with the number of total passengers, as is intuitive. In general, there do not seem to be big differences in the BAT between the doors with and without PEDs in any of the categories. On the first two categories, these differences are lower than $1 \mathrm{~s}$ and favour the absence of PEDs. However, in the third category (when the total boardings and alightings exceed 25 passengers) the average difference of $1.96 \mathrm{~s}$ favours PEDs.

\section{TABLE 2 BAT With (WMS) And Without PEDs (GPK) With Respect To Demand Metrics}

\begin{tabular}{llllllllll}
\multirow{2}{*}{ Metric } & \multicolumn{3}{c}{$\begin{array}{l}\text { Number of } \\
\text { Lbservations }\end{array}$} & \multicolumn{3}{c}{ Mean corrected BAT (s) } & \multicolumn{3}{c}{$\begin{array}{l}\text { Standard deviation of } \\
\text { corrected BAT (s) }\end{array}$} \\
& & GPK & WMS & GPK & WMS & Diff. & GPK & WMS & Diff. \\
\hline Train demand & Low & - & 10 & - & 25.00 & - & - & 9.43 & - \\
on arrival & Medium & 280 & 945 & 19.38 & 21.47 & +2.09 & 6.44 & 6.88 & +0.45 \\
& High & 329 & 748 & 19.33 & 24.94 & +5.61 & 6.37 & 6.96 & +0.60 \\
Total boardings & $0-15$ & 422 & 554 & 17.09 & 17.20 & +0.11 & 5.69 & 5.85 & +0.16 \\
and alightings & $15-25$ & 188 & 809 & 24.26 & 24.46 & +0.20 & 4.76 & 5.42 & +0.65 \\
& $25+$ & 5 & 340 & 31.00 & 29.04 & -1.96 & 4.18 & 5.82 & +1.63
\end{tabular}

\section{Impact on passenger behaviours}

\section{Observed behaviour frequencies}

Figure 2 (right) shows the formation of queues at the hump door in GKP when the train is stopped at the station. Queues beside the doors help passengers alight, reducing the conflict at the PTI.

When the boarding and alighting finishes, another behaviour pattern was observed at the hump door in GKP. In $22 \%$ of the observed trains, passengers preferred to stand on the platform and wait for the next service (135 out of a total of 615 trains). There are two main reasons why a person would decide not to board the train that is just on the platform. One is that there is not enough space available inside for them to feel comfortable, and the other is that their destination station may not be served by the current train, which happens at GPK because some southbound Jubilee line services short-trip a few stations before the last one. However, with the available data, it is impossible to determine what is the true reason in each case.

At the same time, a circulation space was formed between the platform wall and the standing passengers on the platform, where naturally passengers form flow lanes to avoid collisions with people coming in the opposite direction (Figure 2 left).
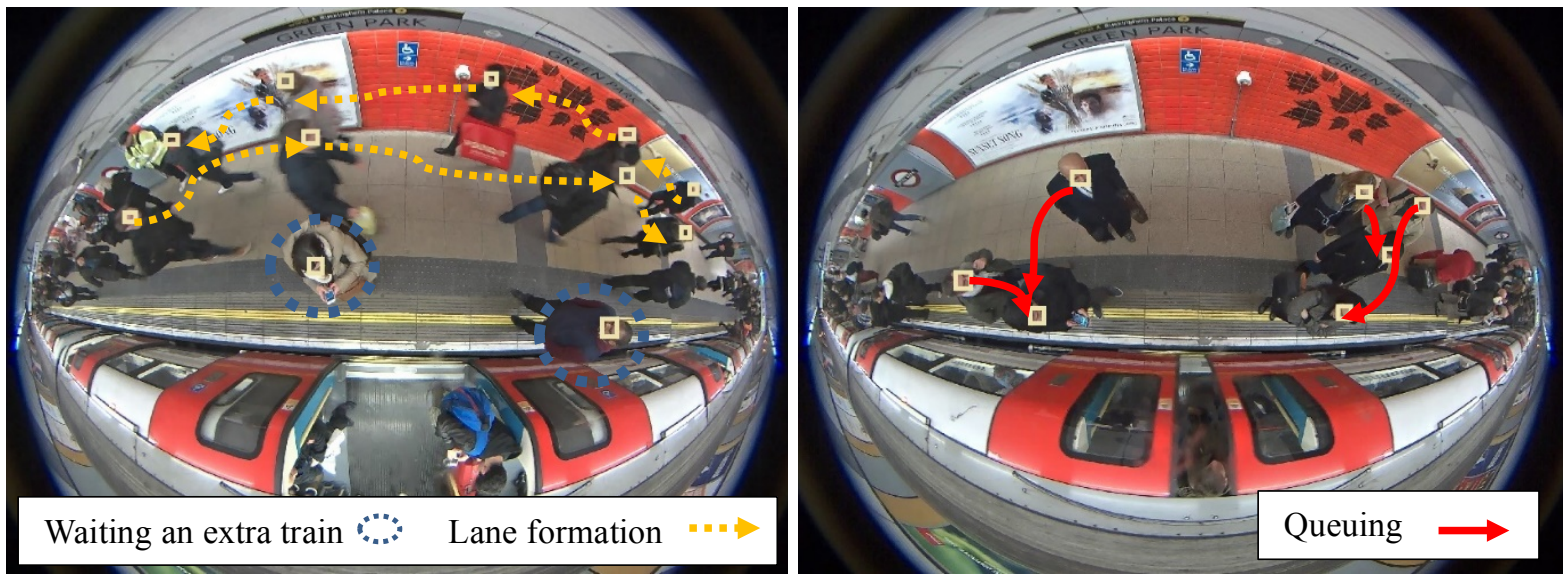


\section{FIGURE 2 Passenger behaviours at the hump door (GPK, no PEDs).}

Figure 3 compares boarding passengers' behaviour between the situation without PEDs (GKP) and the case with PEDs (WMS). The percentages quoted are calculated as the frequency of each behaviour in each category divided by the total number of observations in that category. The data are binned according to the total number of boarders, because people place themselves on the platform based on their surroundings passengers, which has been seen to have an impact on behaviours and this seems to be the best way of capturing that.

For low demand levels (0-15 passengers) the case with PEDs (WMS) presents more passengers waiting in front of the doors than GKP (no PEDs). This behaviour did not change when the demand increased, so that for medium (15-25 passengers) and high (more than 25 passengers) demand levels there were more passengers waiting in front of the doors at WMS (PEDs) than at GKP (no PEDs). In addition, PEDs seem to encourage passengers to wait beside the doors for low and high demand levels, thus reducing the conflict at the PTI, but not for the medium demand situation.

In the case of crowded situations when there are passengers everywhere around the doors, the case with PEDs presents fewer passengers waiting in front and beside the doors for low demand levels. However, this behaviour changed when the level of demand reached medium levels, in which no relevant differences were found between WMS and GKP.

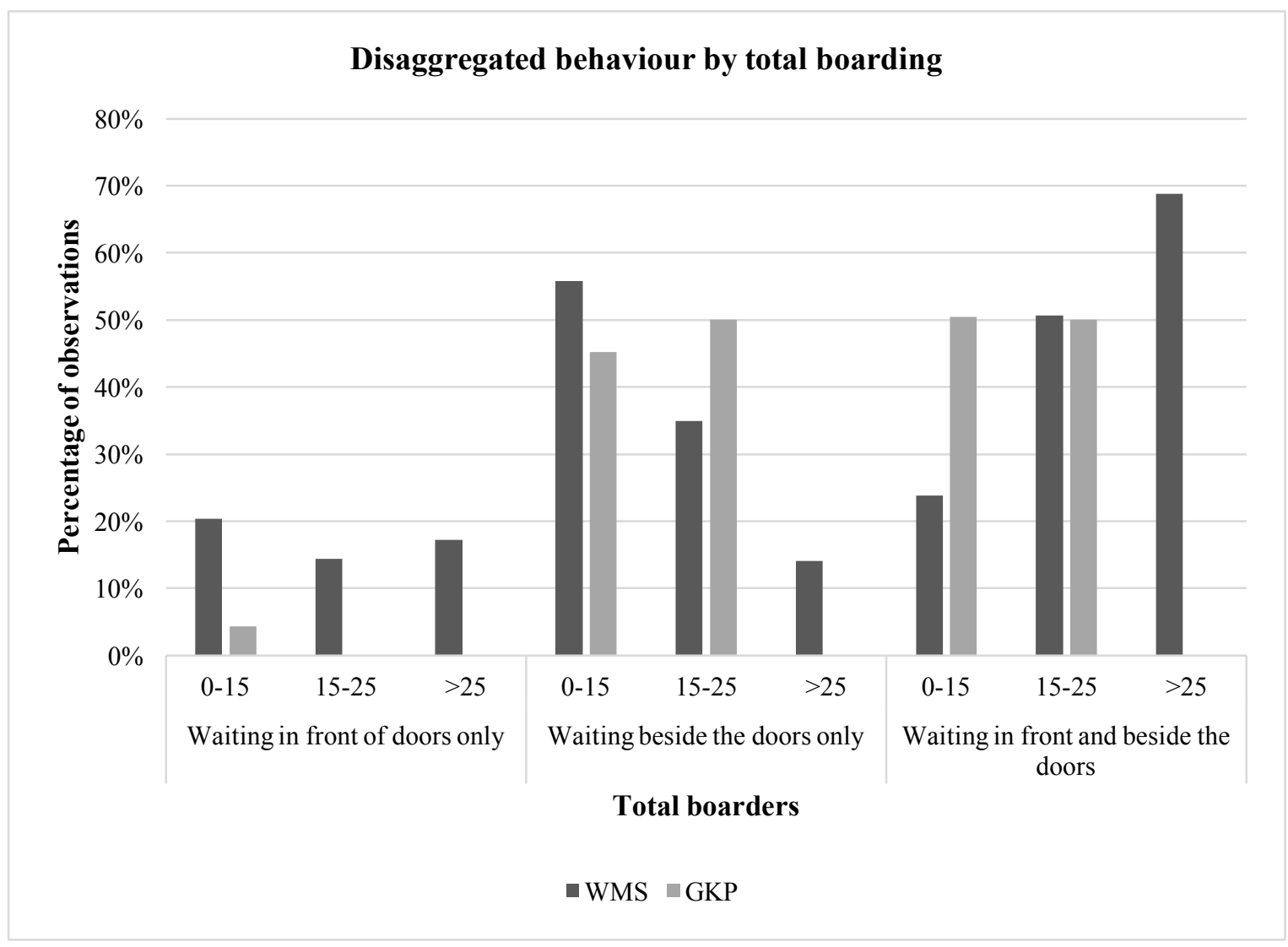

FIGURE 3 Passengers' waiting behaviours with respect to total number of boarders.

\section{Overlap}

The amount of overlap in the boarding and alighting process is another indicator of passengers' behaviour and interactions. At an aggregate level, there seem to be no major differences between PEDs and no PEDs in terms of overlap. However, Figure 4 shows that the average overlap time $\left(T_{\mathrm{o}}\right)$ and the average number of overlaping passengers $\left(\mathrm{P}_{\mathrm{o}}\right)$ changed with respect to the total boarders and alighters. For low (0-15 passengers) and medium (15-25 passengers) demand levels the difference in $T_{o}$ is about $1 \mathrm{~s}$ in favour of PEDs, however this difference reached up to $4 \mathrm{~s}$ for the high demand situation. Similarly, 
with respect to $P_{o}$ no major differences are presented between PEDs and no PEDs for low and medium demand levels, but for the high demand situation this difference reached up to 6 passengers in favour of PEDs.

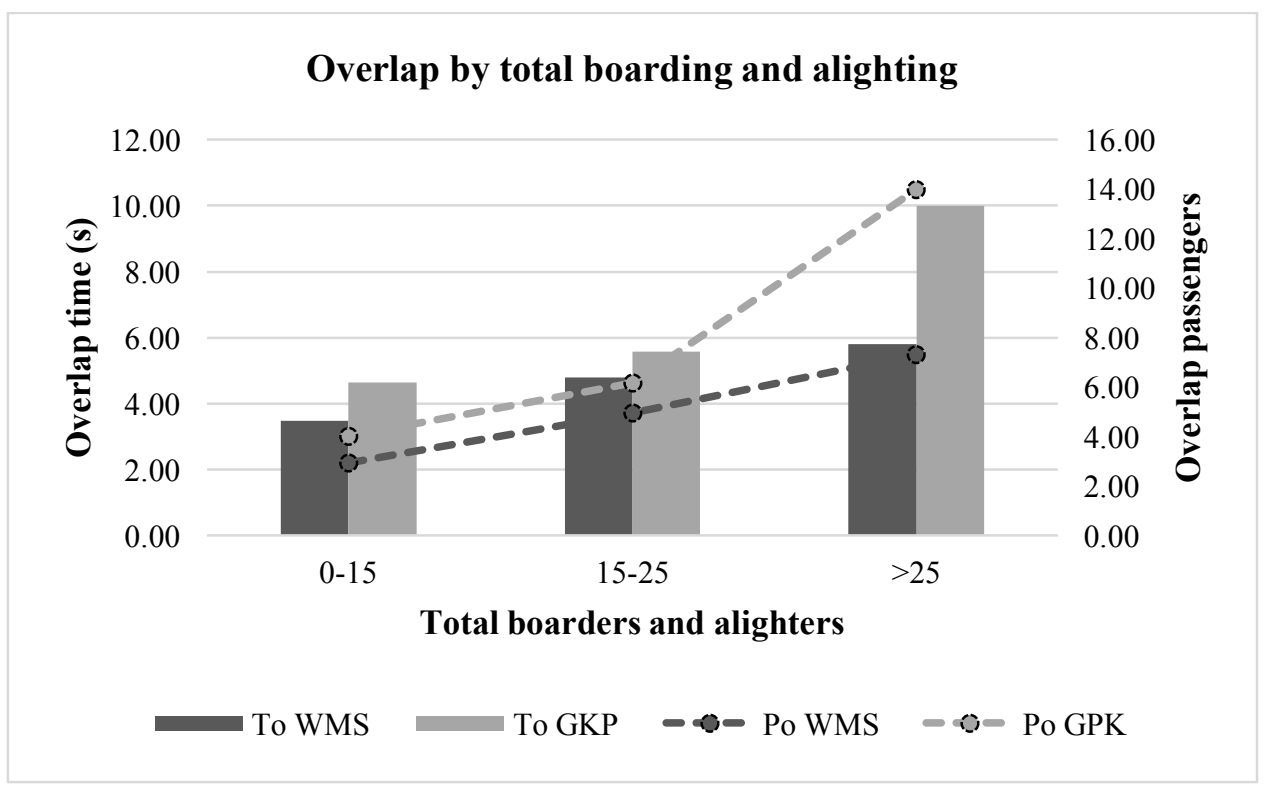

\section{FIGURE 4 Average overlap time and passengers with (WMS) and without PEDs (GPK) with respect to total number of boarders and alighters.}

\section{DISCUSSION}

With respect to the boarding and alighting time (BAT), the case without PEDs (GKP) presents an average BAT which is $16 \%$ lower than in the PEDs case (WMS). This could be interpreted as PEDs increasing the BAT but that would overlook the impact of demand. When demand is taken into account, and similarly to what was found in (6), PEDs not always induce a higher BAT than the case without PEDs. In fact, PEDs only present a BAT that is approximately $1 \%$ higher than the case without PEDs for the first two demand categories of total boarders and alighters (0-15 and 15-25 passengers), but for high demand levels, when the total number of boarders and alighters exceeds 25, PEDs have a BAT that is $7 \%$ lower than the case without PEDs, i.e. PEDs seem to be more effective in dealing with high levels of crowding.

Although at GKP (no PEDs) the average relative boarding profile presents an earlier and higher peak compared to WMS (PEDs), in both cases the profiles converge after $32.5 \mathrm{~s}$, which is the time when most of the boarding is finished. Something similar occurs with the average relative alighting profiles. Therefore, from the point of view of these profiles, there is no impact of PEDs on the BAT.

All in all, and bearing in mind the methodological limitations, there seems to be no overall negative impact of PEDs on the BAT, in line with what was found in (6) and opposed to the preconceived concern. In fact, there seems to be a minor advantage of PEDs in crowded situations.

With regards to passenger behaviour at the platform, there seem to be two distinct regions: a circulation and a waiting area.

The circulation area appears at the back of the platform, near the wall and parallel to it, when passengers form flow lanes to avoid collisions with people coming in opposite direction. Further research is needed to study the relationship between the formation of lanes and the presence of PEDs.

The behaviour in waiting areas is dominated by the boarding passengers who are waiting on the platform. As opposed to what was found in (6), the presence of PEDs does not always change passenger behaviour. PEDs seem to have an important effect in encouraging passengers to wait beside the doors for low (less than 15 boarders) and high (more than 25 boarders) demand levels, but not for a medium levels. Conversely, PEDs have a positive impact on preventing passenger to wait in front and beside the doors for low demand levels, which could be used to control crowded situations.

To correctly interpret this analysis, it should be noted that the results are influenced by the ratio between boarders and alighters, which in the case without PEDs is $63 \%$ smaller than in the case 
with PEDs. Therefore, the detailed level of demand and the exact position of each passenger should be included in further research as factors that influence behaviour.

Another behaviour was observed, whereby some passengers stayed on the platform even when there was a train and waited for the next one. This could be done to either overcrowding on the train at the boarding point or because the train destination does not match the passenger's destination. However, we lack data to assess this impact in detail.

In relation to the boarding and alighting dynamics, the results are in accordance with (11), where alighting occurs before boarding, first at a higher speed and then slowed down due to the increasing interaction with boarding passengers. This interaction (or overlap) was found to be smaller in the case with PEDs in (6), but in this study the reduction was found to be negligible for the low and medium demand levels. In the high demand situation the case with PEDs reached $42 \%$ less overlap time and $48 \%$ fewer overlap passengers than the case without PEDs. These results are in line with what was found in (6), whereby the presence of PEDs is related to less overlap, possibly because PEDs induce a more organised boarding and alighting process with less friction, where boarders tend to give way to alighters more often.

\section{CONCLUSIONS}

This paper has studied the combined effect of platform edge doors (PEDs) and level access on the boarding and alighting time (BAT) and passenger behaviour by means of observation at two LU stations. The results show that there is no clear advantage or disadvantage of PEDs versus no PEDs in terms of BAT, where demand plays a more important role. In any case, there seems to be no clear negative impact of PEDs on BAT (as opposed to what is normally believed) and even PEDs could be minorly beneficial in crowded situations.

With respect to passenger behaviour, the results show that the presence of PEDs influences passenger behaviour and encourages waiting beside the doors rather than in front of them, probably because passengers can see where the doors are. However, this only happens at certain levels of demand.

Further research should deepen the analysis of the combined effects of demand and the PTI characteristics and also look at the relationship between the BAT and different vertical/horizontal gaps, because in this case two situations with level acess have been compared. It would also be interesting to collect more data to identify the impact of passengers with encumbrances (luggage, shopping, buggy) or mobility aids (wheelchair, pram) on the BAT. 
1 ACKNOWLEDGEMENT

2 The authors would like to thank Transport for London for its support and for providing the data for this 3 study. 


\section{REFERENCES}

1. Law, C. K., and P. S. Yip. An economic evaluation of setting up physical barriers in railway stations for preventing railway injury: evidence from Hong Kong. Journal of epidemiology and community health, Vol. 65, No. 10, 2011, pp 915-920.

2. Kyriakidis, M., R. Hirsch, and A. Majumdar. Metro railway safety: An analysis of accident precursors. Safety Science, Vol. 50, No. 7, 2012, pp 1535-1548.

3. Kroes, E., M. Kouwenhoven, L. Debrincat, and N. Pauget. Value of Crowding on Public Transport in Île-de-France, France. In Transportation Research Record: Journal of the Transportation Research Board, No. 2417, Transportation Research Board of the National Academies, Washington, D.C., 2014, pp 37-45.

4. Guidance on Interface between Station Platforms, Track and Trains, Rail Industry Guidance Note for GI/RT7016, Rail Safety and Standards Board, London, 2014.

5. Taking forward the Mayor's Transport Strategy Accessibility Implementation Plan. Transport for London, 2012. http://content.tfl.gov.uk/taking-forward-the-mts-accessibilityimplementation-plan-march-2012.rtf. Accessed April 25, 2016.

6. De Ana Rodríguez, G., S. Seriani, C. Holloway. The impact of platform edge doors on passengers boarding and alighting time and platform behaviour. Proceeding of the Transportation Research Board 96th Annual Meeting, 2016. Accepted for publication in Journal of the Transportation Research Board, January 30, 2016.

7. Management of on-train crowding, Final Report, Rail Safety and Standards Board. London, 2008.

8. Highway Capacity Manual 2000, Special Report 209, Transportation Research Board, Washington D.C., 2000.

9. Transit Capacity and Level of Service Manual, 2nd Edition, TCRP Report 100, Transportation Research Board, Washington D.C., 2003.

10. Wiggenraad, B.L. Alighting and Boarding Times of Passengers at Dutch Railway Stations. Research Report, Delft University of Technology, 2001.

11. Harris, N. G. Train boarding and alighting rates at high passenger loads. Journal of advanced transportation, Vol. 40, No. 3, 2006, pp 249-263.

12. Harris, N. G., and R. J. Anderson. An international comparison of urban rail boarding and alighting rates. Proceedings of the Institution of Mechanical Engineers, Part F: Journal of Rail and Rapid Transit, Vol. 221, No. 4, 2007, pp 521-526.

13. Fernandez, R., P. Zegers, G. Weber, and N. Tyler. Effect of Door Width, Platform Height and Fare Collection on Bus Dwell Time. Laboratory evidence for Santiago de Chile. In Transportation Research Record: Journal of the Transportation Research Board, No. 2143, Transportation Research Board of the National Academies, Washington, D.C., 2010, pp 59-66.

14. Seriani, S., T. Fujiyama, C. Holloway. Pedestrian level of interaction on platform conflict areas by real-scale laboratory experiments. Proceeding of the 48th Annual University Transport Study Group Conference, 2016, Bristol.

15. Qu, L., and W. K. Chow. Platform screen doors on emergency evacuation in underground railway stations. Tunnelling and Underground Space Technology, Vol. 30, 2012, pp 1-9.

16. Allen, S. An integrated systems engineering approach to managing the implementation of new transit technologies. Proceedings of the In Railroad Conference 1995 IEEE/ASME Joint, Toronto.

17. New tube for London. Feasibility Report. London Underground Limited, 2014. http://content.tfl.gov.uk/ntfl-feasibility-report.pdf. Accessed April 26, 2016.

18. Holloway, C., R. Thoreau, T.-R. Roan, D. Boampong, T. Clarke, and D. Watts. Effect of Vertical Step Height on Boarding and Alighting Time of Train Passengers. Proceedings of the Institution of Mechanical Engineers Part F Journal of Rail And Rapid Transit, 2015, DOI: 10.1177/0954409715590480

19. Heinz, W. Passenger service times on trains-theory, measurements and models. Ph.D. Thesis, Royal Institute of Technology, Stockholm, Sweden, 2003.

20. Tyler, N., C. Childs, D. Boampong, T. Fujiyama. Investigating ramp gradients for humps on railway platforms. Proceedings of the Institution of Civil Engineers - Municipal Engineer, Vol. 168, No. 2, 2015, pp 150-160. 
21. Fernandez, R., A. Valencia, and S. Seriani. On Passenger Saturation Flow in Public Transport Doors. Transportation Research Part A, Vol. 78, 2015, pp 102-112.

22. Fujiyama, T., R. Thoreau, and N. Tyler. The effects of the design factors of the train-platform interface on pedestrian flow rates. Pedestrian and Evacuation Dynamics 2012, Springer International Publishing, 2014, pp 1163-1173.

23. Daamen, W., Y. Lee, and P. Wiggenraad. Boarding and Alighting Experiments: An Overview of the Set Up and Performance and Some Preliminary Results on the Gap Effects. In Transportation Research Record: Journal of the Transportation Research Board, No. 2042, Transportation Research Board of the National Academies, Washington, D.C., 2008, pp 71-81.

24. Karekla, X., and N. Tyler. Reduced dwell times resulting from train-platform improvements: the costs and benefits of improving passenger accessibility to metro trains. Transportation Planning and Technology, Vol. 35, No. 5, 2012, pp 525-543. 